\title{
MR and CT Imaging Spectrum of Right-Sided Cardiac Masses: A Pictorial Essay
}

\author{
Murugan MK, Gulati GS
}

\begin{abstract}
Department of Cardiovascular Radiology, All India Institute of Medical Sciences, Ansari Nagar, Delhi-110029, India
\end{abstract}

\section{Introduction}

Primary cardiac neoplasms are rare and are encountered in approximately 0.02 to $0.1 \%$ of autopsy cases. ${ }^{1}$ Primary cardiac neoplasms are more commonly benign (79$85 \%$ ) than malignant. ${ }^{2,3,4}$ However, metastases are by far the most common cardiac neoplasmswith a reported incidence of $1.2 \% .^{5,6}$ Including all cardiac masses, thrombus is the most common entity followed by metastasis. While myxomas and sarcomas account for most tumors in adults, teratomas and rhabdomyomas are most frequently found in infants and fibromas are most common in children. The most common clinical symptoms of patients with a cardiac neoplasm are due to heart failure followed by thromboembolic events. The latter are characteristically the initial clinical manifestation for myxoma. ${ }^{2}$ Multiple imaging modalities are available for evaluation and characterization of primary and secondary tumors involving the heart. Cross-sectional imaging with cardiac CT (CCT), cardiac MR (CMR), and 18F-FDG $\mathrm{PET} / \mathrm{CT}$ work synergistically with

Corrospondence to: Gurpreet Singh Gulati,

Additional Professor, Cardiovascular Radiology, Cardiothoracic Centre, All India Institute of Medical Sciences, Ansari Nagar, Delhi-110029, India, Tel: 91-11-26594452 Email: gulatigurpreet@ rediffmail.com echocardiography. Echocardiography is typically the primary modality used for diagnosis or evaluation of a suspected cardiac mass because of its availability, noninvasiveness, absence of contrast material or radiation exposure, and ability to make dynamic assessment of cardiac masses.

However it lacks in specificity, technical difficulty in selected population like obese individuals, global assessment and soft tissue characterization. $^{7}$

CMR is the most important modality in differentiating tumor from thrombus and distinguishing benign from malignant cardiac masses, and it helps to determine the ex-tent of myocardial and pericardial invasion in cardiac masses. ${ }^{8}$ The usefulness of CMR includes its ability to detect the neoplasm, define its precise location, border and impingement on surrounding structures,characterize the lesion contents through tissue characterization, estimate the effectiveness of chemotherapy and radiotherapy, and differentiate malignant from benign tumors.

CCT has a limited role in tissue characterization as compared to the CMR imaging. But the global information available is valuable in formulating the differential diagnosis, like the associated 

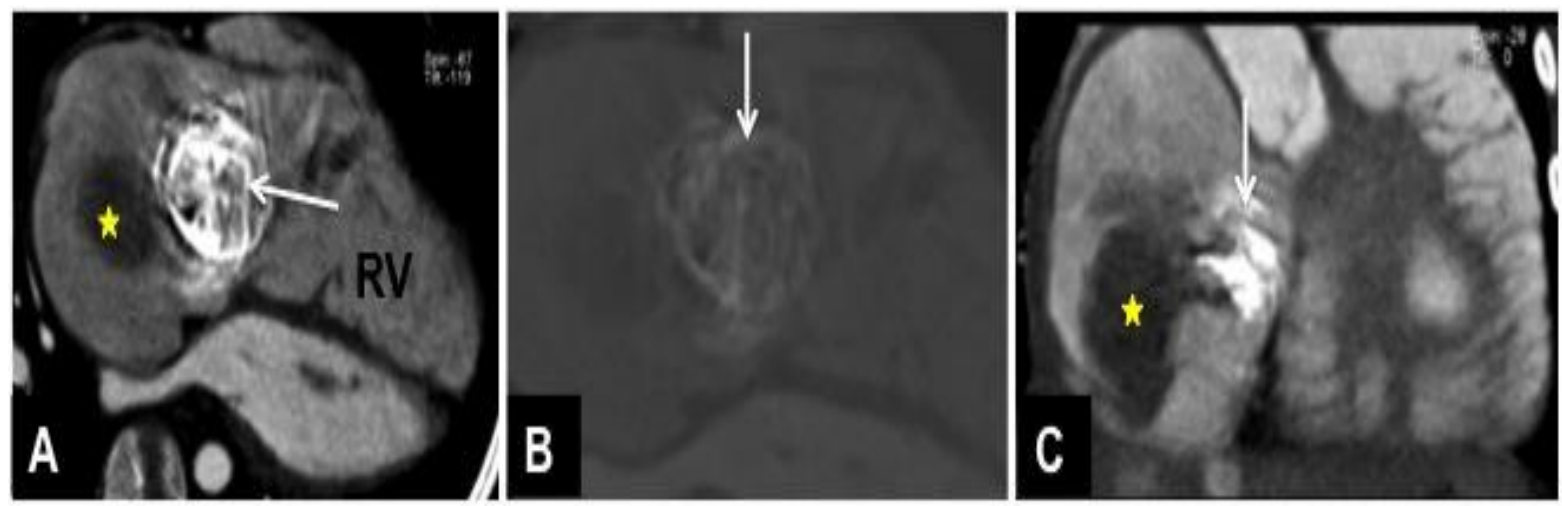

Fig 1: Myxoma in a 10year old boy with dyspnea and palpitation. (A) $4 \mathrm{CH}$ view of CTA and (B) 4CH view bone window, showing a large hypo dense mass (yellow star) with area of chunky calcification (white arrow), attached to the tricuspid valve and projecting in to the RA. (C) VLA view showing the mass in relation to the tricuspid valve.
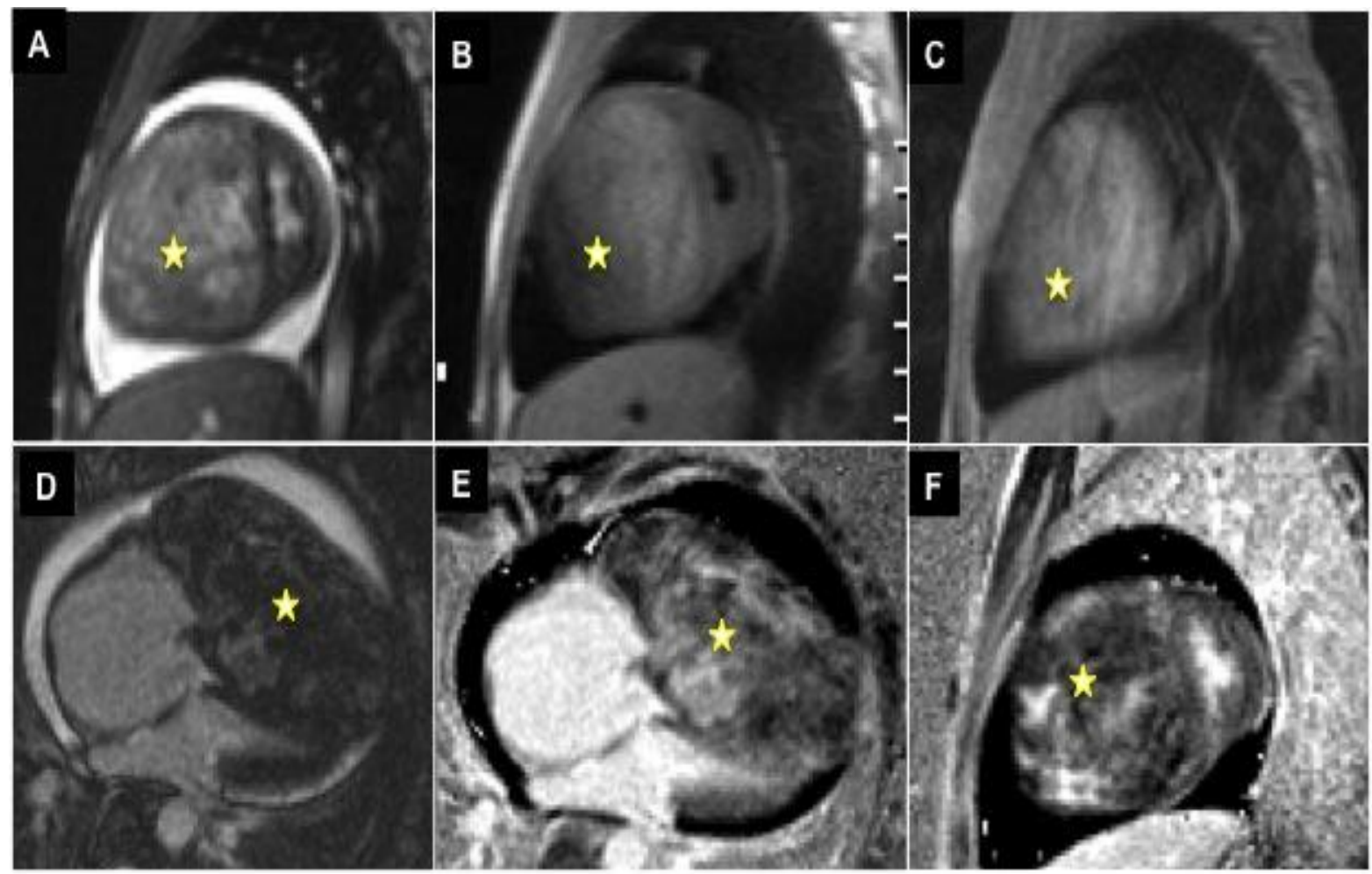

Fig 2: Rhabodmyosarcoma in a 6year old boy: (A) SSFP SA view show large heterogenous mass (yellow star) arising from the RV wall, causing expansion and replacement of RV with mass effect. Pericardial effusion is present. (B \& C) T1W and T2 FS images in SA view show signal character of lesion, which is heterogeneously iso to intermediate in T1 and hyper on T2 weighting. Post LGE images (D, E) 4CH and (F) SA views show the heterogenous enhancement of the RV mass lesion. 


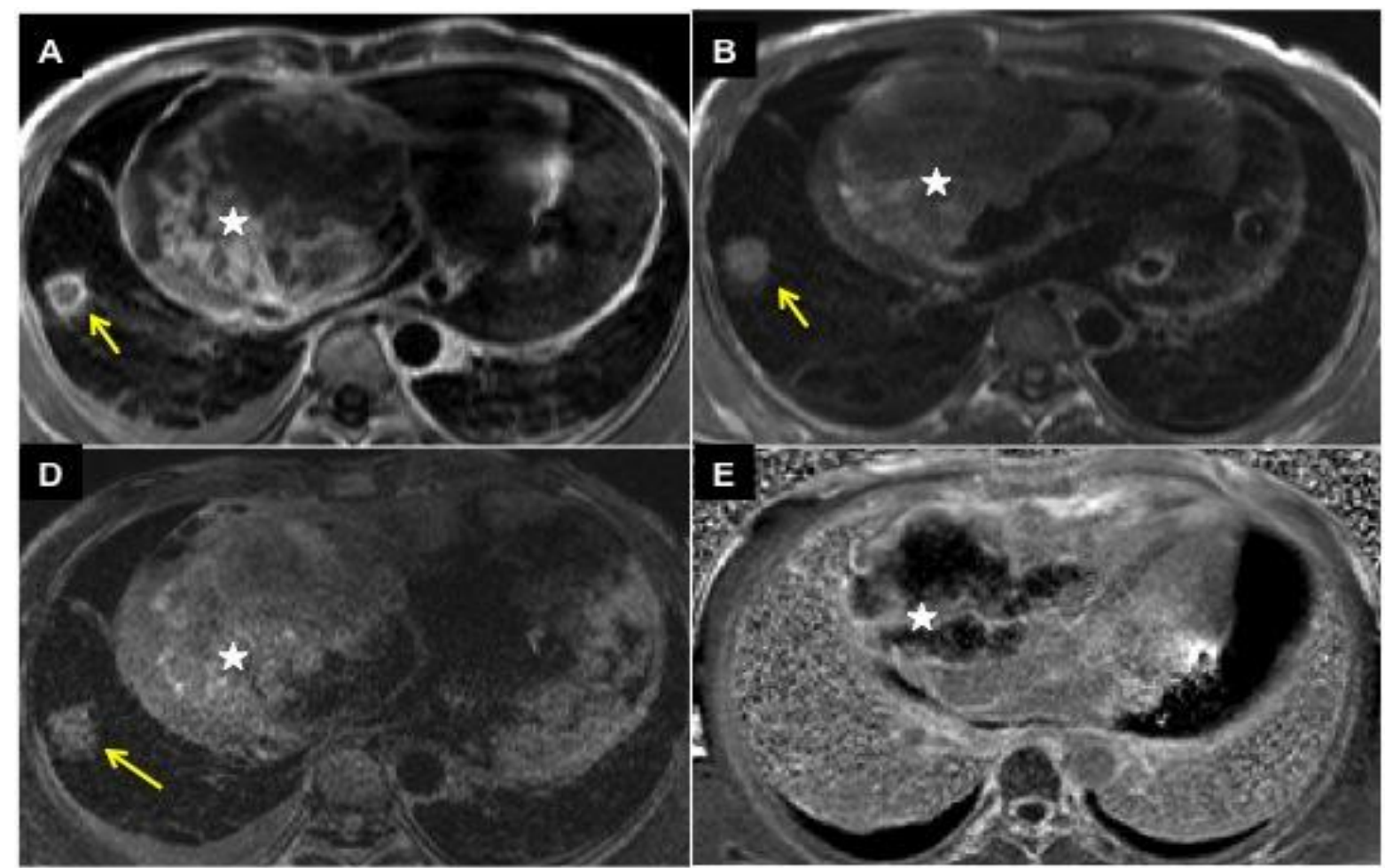

Fig 3: Angiosarcoma with lung metastasis: (A) HASTE axial show large heterogenous mass (white star) arising from RA and crossing the Tricuspid valve to reach the RV. Nodular lesion seen in the right lower lobe (yellow arrow) of the lung noted (B, C) T1 and T2 FS axial images show the signal character of the lesion which is heterogeneously iso to intermediate on T1 and intermediate to hyper on T2. (D) LGE inversion recovery sequence axial section shows the heterogenous enhancement with multiple nonenhancing necrotic areas.

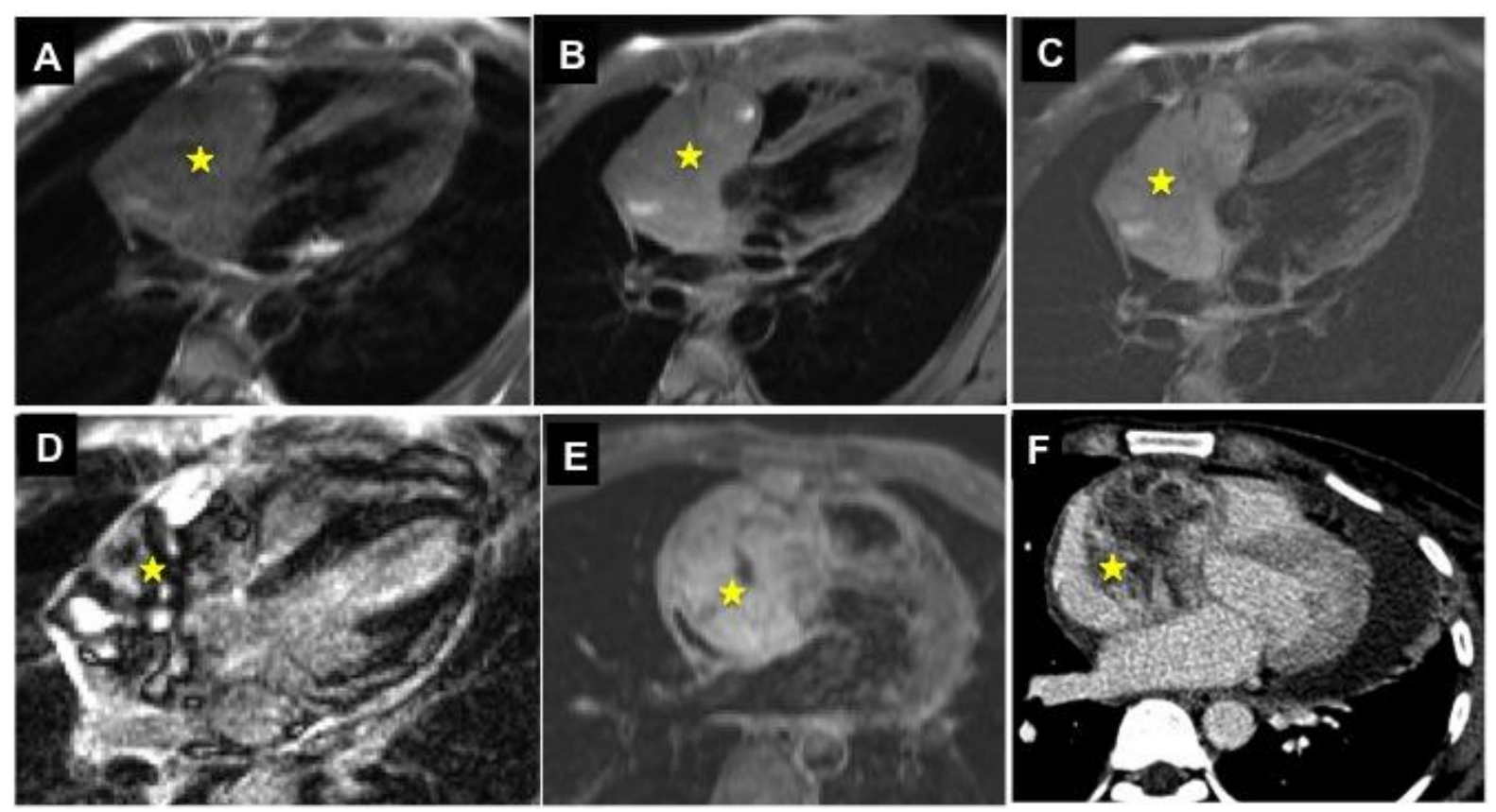

Fig 4: Malignant mesothelioma: (A) T1W 4CH view show heterogeneously intermediate signal mass (yellow star) with few areas of high signal intensity which are not 
suppressed on fat suppression (B) T1 FS 4CH image. (C) T2W 4CH view shows diffusely heterogeneous high SI. (D) 4CH inversion recovery LGE sequence also shows heterogenous enhancement along with infiltration of the RA and RV myocardium. (E)T1 FS Post contrast axial image show intense contrast uptake by the lesion with few enhancing necrotic areas. (F) Axial CT section showing enhancing necrotic right-sided mass with pericardial effusion.
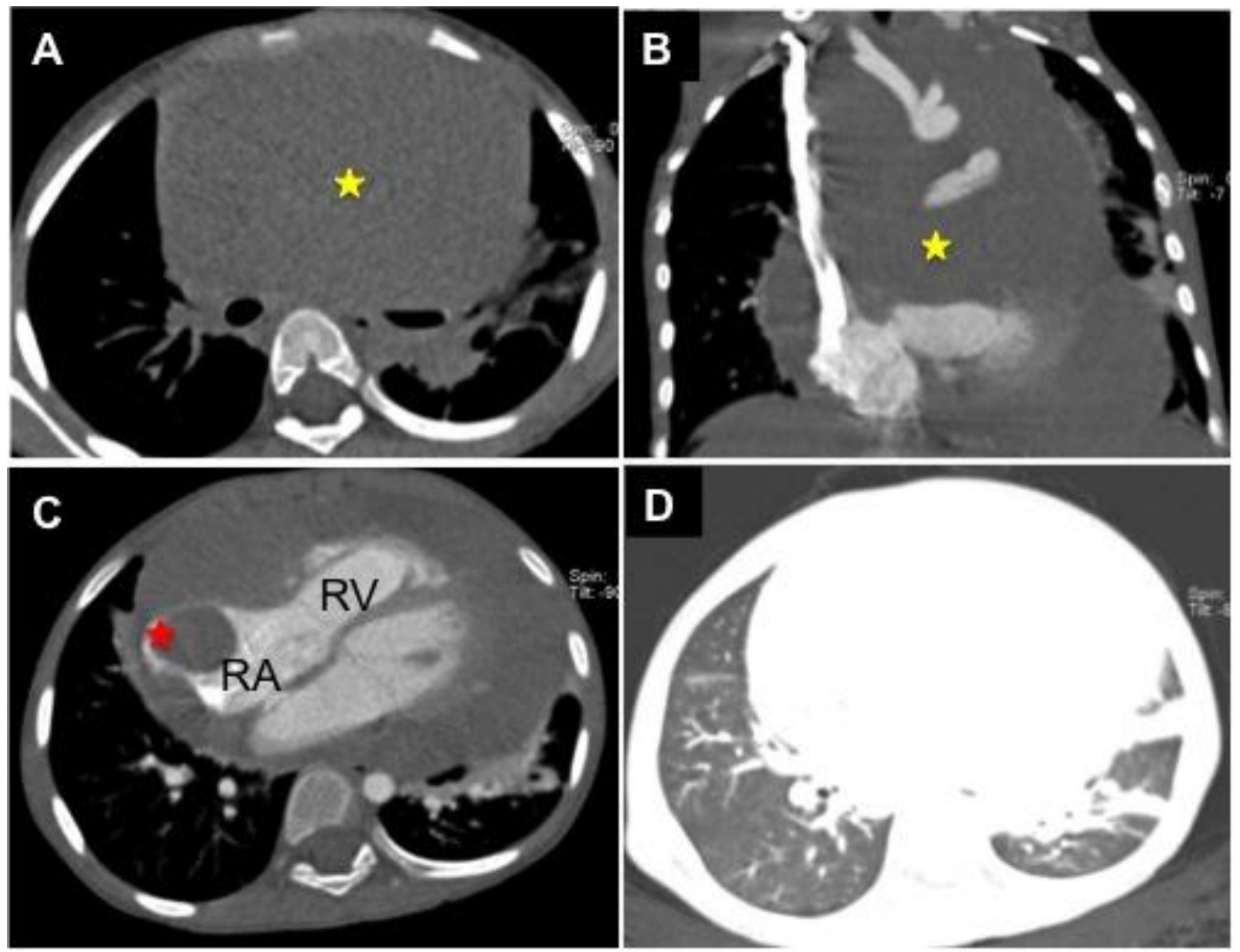

Fig 5: Lymphomatous deposit in an 8-year female child with NHL, involvement of mediastinum, pericardium and RA. (A) NCCT axial section shows presence of homogenous sheet like soft tissue. No calcification or fat seen. (B) Contrast enhanced gated CT coronal and (C) axial section shows the mass is encasing the mediastinal structure and also indentation of the Right AVG and RA. Minimal contrast uptake is seen. There is polypoidal mass (red arrow) protruding into the RA. Pericardial effusion is also present. No necrosis seen. (D)Axial CT lung window shows patchy atelectasis in left lower lung. No nodules present. 
lung involvement, other metastasis, lymph nodes, calcification identification, etc.

In this article, we discuss the CMR and CCT imaging features in selected right sided cardiac masses and review the characteristic imaging findings of specific conditions.

\section{Clinical Experience}

Over a 14-year period, 58 patients were analyzed retrospectively, in whom CMR and/or CCT was performed at our institute following suspicion of right sided mass on ECHO. Thirty-three patients underwent CMR (1.5 Tesla, Magnetom, Siemens, Germany; or 3 Tesla, Achieva, Philips, Netherlands), 16 patients (all patients too sick for CMR) had CCT (64-slice dual source, Somatom Definition, Siemens, Germany), whereas 9 patients had both CCT and CMR. CMR protocol included Spinecho T1-weighted (T1W) (with \& without fat suppression, FS), T2-weighted (T2W) (FS), Gradient recalled echo (GRE) T2W, HASTE, CINE, Perfusion, Inversion recovery Late gadolinium enhancement (LGE), T1 W FS post gadolinium, Phase Contrast (PC) (where needed). CMR sequences performed in cardiac specific planes. CCT protocol included non-gated / gated non-contrast $\mathrm{CT}$ of limited coverage section, gated cardiac CT angio in arterial phase, delayed non-gated study with coverage of entire thorax was performed with retrospective ECG gating. Imaging findings were analyzed for possible etiology, location, extent, hemodynamic effects and extra-cardiac manifestations.

\section{Discussion}

Lesions presenting as right sided cardiac masses may fall broadly in to either of the category, neoplastic or non-neoplastic. Neoplastic masses may be primary or secondary. Benign primary cardiac neoplasms include myxoma, fibroma, rhabdomyoma, papillary fibroelastoma, hemangioma, paraganglioma, lipoma and malignant primary neoplasm include sarcomas like angiosarcoma, rhabdomyosarcoma, fibro sarcoma, synovial sarcoma, mesotheliomas and lymphomas. Among the malignant etiology secondary metastasis accounts for the most common cause $^{9}$ and it may be due to direct venous invasion (renal cell carcinoma or hepatocellular carcinoma), direct contiguous invasion from a right side lung carcinoma or breast carcinoma, or a hematogenous seeding from distant primary or lymphoma.

Among the non-neoplastic causes of cardiac masses, thrombus accounts for most of the cases. Other non-neoplastic causes encountered in the differentials of right-sided cardiac masses include infective/inflammatory masses, vascular lesions and pseudo tumors. Tubercular masses like tuberculoma or tubercular abscess accounts for the most common infective right-sided cardiac mass, and it was the most common right-sided cardiac mass in our observation followed by malignant etiology.

Pericardial masses presenting as right-sided lesions include pericardial abscess / lobulated effusion, pericardial cyst and primary or metastatic pericardial tumors. 

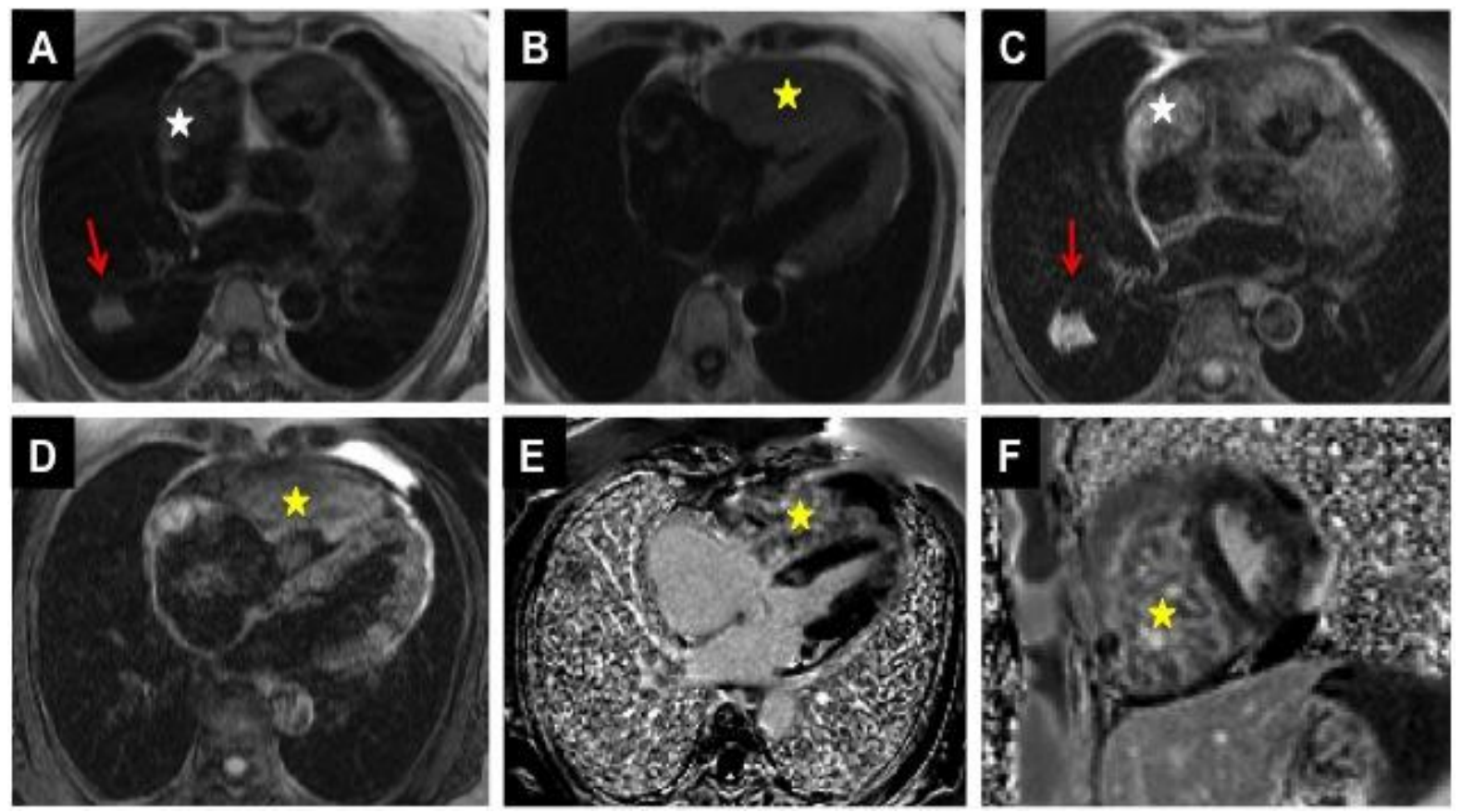

Fig 6: Metastasis- Hematogenous - from a postoperative renal cell carcinoma. (A, B) axial T1 W images show presence of mass in the RA appendage (white star) and RV myocardium (yellow star), which show iso intense signal. $(C, D)$ in axial T2 W image the lesions are heterogeneously hyper intense. In addition nodular lesion is seen in the right lower lobe of lung (red arrow). (E, F) 4CH and SA LGE inversion recovery images show the heterogenous enhancement of the lesion.
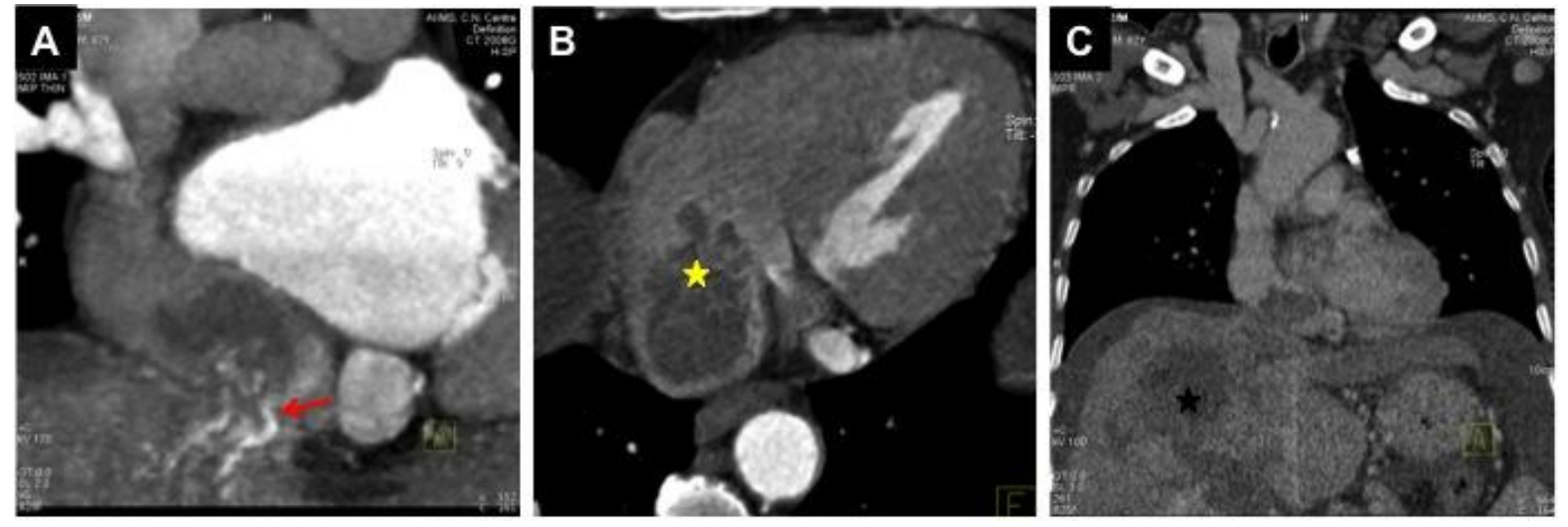

Fig 7: Metastasis - from Hepatocellular carcinoma with direct vascular invasion through Inferior vena cava into the RA. CT angiographic images (A) oblique MIP reformat showing direct invasion of thrombus in the $\mathrm{RA}$ with serpiginious hyper dense vessels (red arrow) within it which are seen in continuity from the left lobe liver lesion. (B)axial section shows the enhancing mass (yellow star) in the RA - IVC junction region. (C) Delayed contrast enhanced CT coronal section showing the wash out in the liver lesion (black star) and the direct vascular invasion of the RA from the liver lesion. The liver surface is irregular and there is presence of ascites. Suggesting the underlying chronic liver disease. Alpha feto protein was very high. 


\section{Neoplastic Etiology}

\section{Benign primary cardiac neoplasm}

\section{Myxoma}

The most common site of cardiac myxoma is LA (75\%), followed by RA (20\%) and rarely seen in the ventricles ${ }^{10}$ Myxomas may present as a homogenous mass with iso intense signal as that of myocardium on $\mathrm{T} 1$ and hyper on $\mathrm{T} 2$ with low to high post gadolinium enhancement. Presence of calcification, hemorrhage, and necrosis within the mass makes it appear more heterogenous on T1 SE, T2 SE and GRE sequences. Cardiac CT helps in identification of the calcification (Fig 1). Hypointensity and pedicle are better demonstrated in cine SSFP sequences with CMR. ${ }^{11,12}$ On perfusion imaging hypoperfusion is reported with cardiac myxoma. ${ }^{13}$

\section{Rhabdomyoma}

They are the most common benign cardiac neoplasm in children. Nearly $50 \%$ of the rhabdomyomas are syndromic and associated with tuberous sclerosis. ${ }^{14}$ Often they are multiple with variable sizes, sometimes they are tiny and manifest as diffuse myocardial thickening. Rhabdomyomas are iso or hyperintense to myocardium on $\mathrm{T} 1 \mathrm{w}$ images and slightyhyperintense on $\mathrm{T} 2 \mathrm{w}$ images. ${ }^{12}$ The borders of the tumours are better identified on proton density weighted images and post contrast images. ${ }^{11}$

\section{Fibroma}

This is the second most common benign cardiac neoplasm in children. They are often seen in the inter ventricular septum and in the LV myocardium and typically have a lower signal intensity on T2 SE compared with other tumors due to the fibrous content. $^{15}$

\section{Lipoma}

These are encapsulated masses and parallel the fat signal in all sequences and show a characteristic hyper intense signal on $\mathrm{T} 1 \mathrm{~W}$ SE, which are suppressed on fat suppression sequences. ${ }^{15}$ Cardiac lipomas are distributed throughout the heart in endocardial, myocardial, and epicardial locations. ${ }^{16}$ Due to the typical signal characteristics contrast administration is usually not required to make the diagnosis of lipoma, except in cases where a sarcomatous change is suspected.

\section{Malignant primary cardiac neoplasm}

\section{Sarcomas}

Sarcomas are the most common primary cardiac malignancy and the second most common primary cardiac tumor after myxoma. ${ }^{15}$ They are rare mesenchymal tumors and the most common subtype is an angiosarcoma. $^{10}$

\section{Rhabdomyosarcoma}

They usually occur in infants and young children with no definite chamber predilection. ${ }^{10}$ They are typically intramural and have a tendency to arise from the valves 17 They appear heterogeneously iso -hyper on T1, hyper on $\mathrm{T} 2$ and show inhomogenous enhancement with large areas of central necrosis (Fig 2). Secondary involvement of the pericardium may be seen. ${ }^{18}$ 

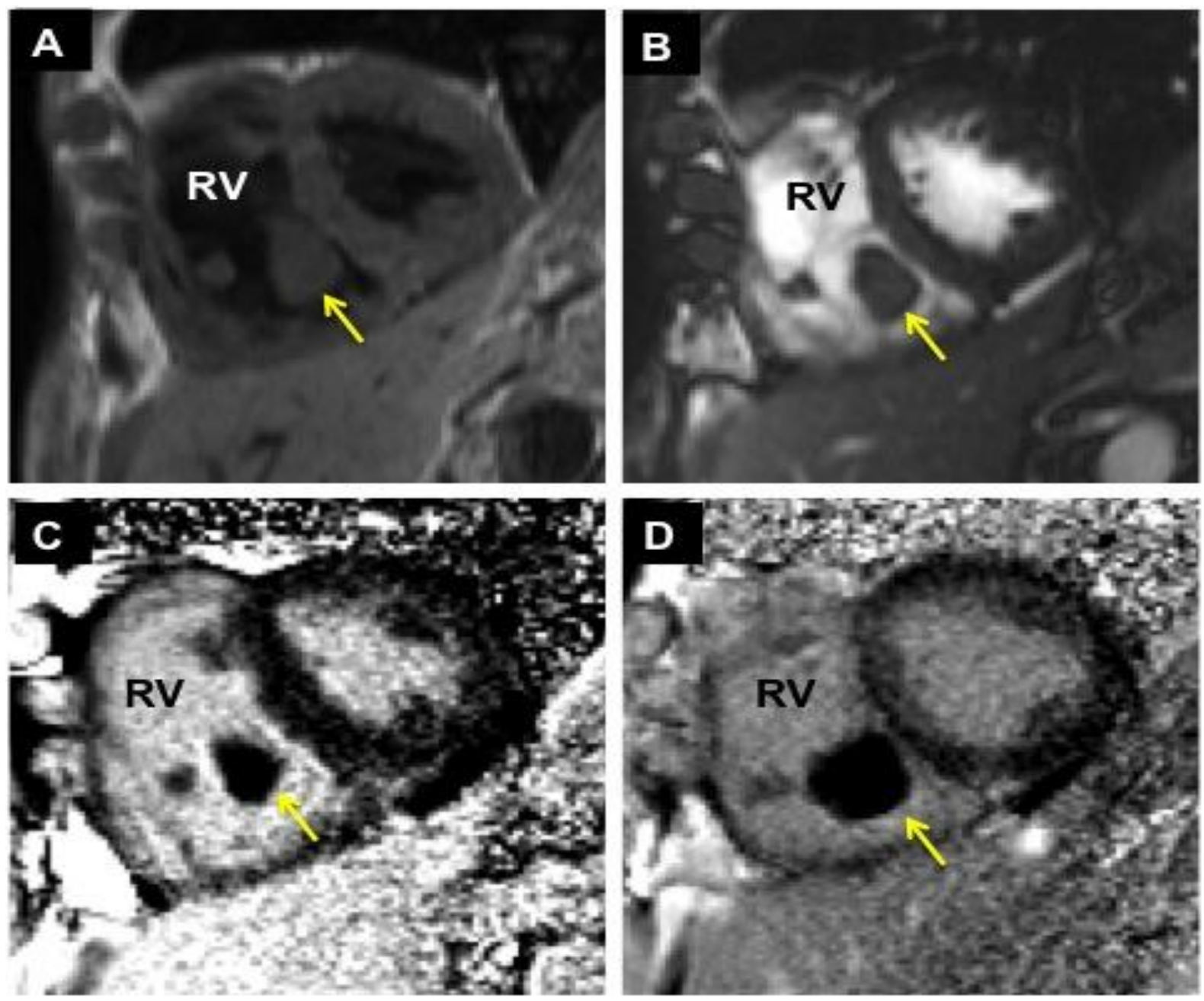

Fig 8: RV Thrombus: (A) T1 W SA view showing iso intense mass (yellow arrow) in RV cavity. (B) TRUFI sequence SA shows the mass distinctly in the $R V$, which is iso intense in signal. ( $C$ and $D)$ Post gadolinium inversion recovery sequence with increasing inversion time (TI $250 \mathrm{~ms}, 400 \mathrm{~ms}$ respectively) shows no evidence of any contrast enhancement.

\section{Angiosarcoma}

Angiosarcomas usually occur in adult male population. The most common site is RA, although they can extend to the pericardium or sometimes originate in pericardium in patients with Kaposi's sarcoma. ${ }^{10}$ They are markedly heterogenous in $\mathrm{T} 1$ and $\mathrm{T} 2 \mathrm{~W}$ images (Fig 3). Areas of intratumoral hemorrhage appear as hyperintense foci on SE images and hypointense on gradient echo images. Hyperintense areas in GRE sequences correspond to flow voids from vascular structures are also seen. This feature has been described as a "cauliflower" appearance $^{11}$. Angiosarcomas typically show diffuse and intense contrast enhancement that has been described as a "sun- ray" appearance. $^{19}$

\section{Mesothelioma}

Mesothelioma arises from pericardium and may or may not secondarily invade the 

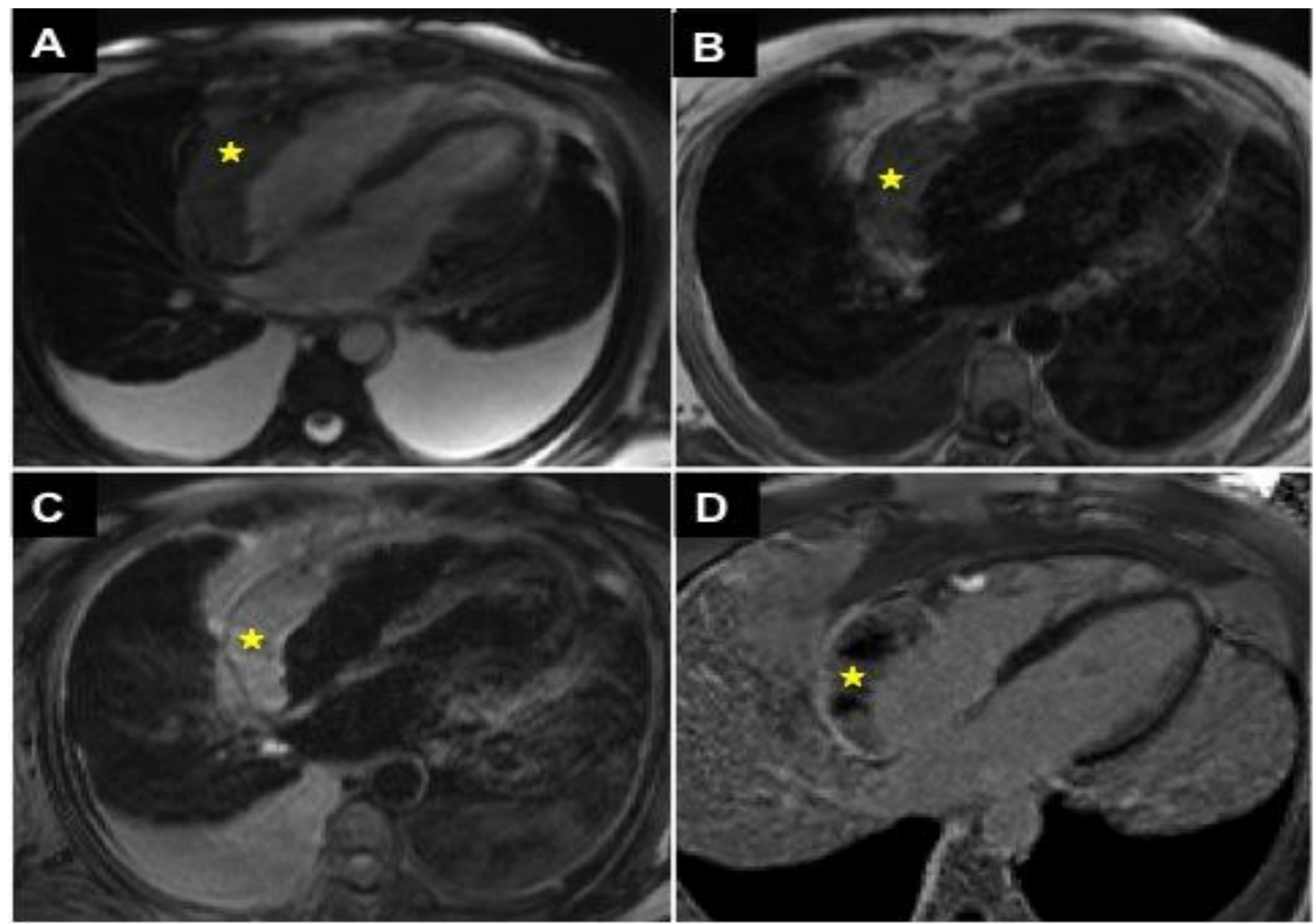

Fig 9: Tubercular Mass 30-year-old female. (A) Axial SSFP image show presence of a nodular soft tissue mass (yellow star) in the right side of the heart involving the RA. Bilateral pleural effusion present. (B, C) T1 W and T2 W FS 4CH view respectively, shows heterogenous iso to hypo intense signal on $\mathrm{T} 1$ and intermediate to high SI on T2. (D) $4 \mathrm{CH}$ view post gadolinium inversion recovery sequences show the heterogenous signal enhancement of the lesion with few non-enhancing areas within it. Biopsy from the lesion showed granulomatous inflammation consistent with tuberculosis.
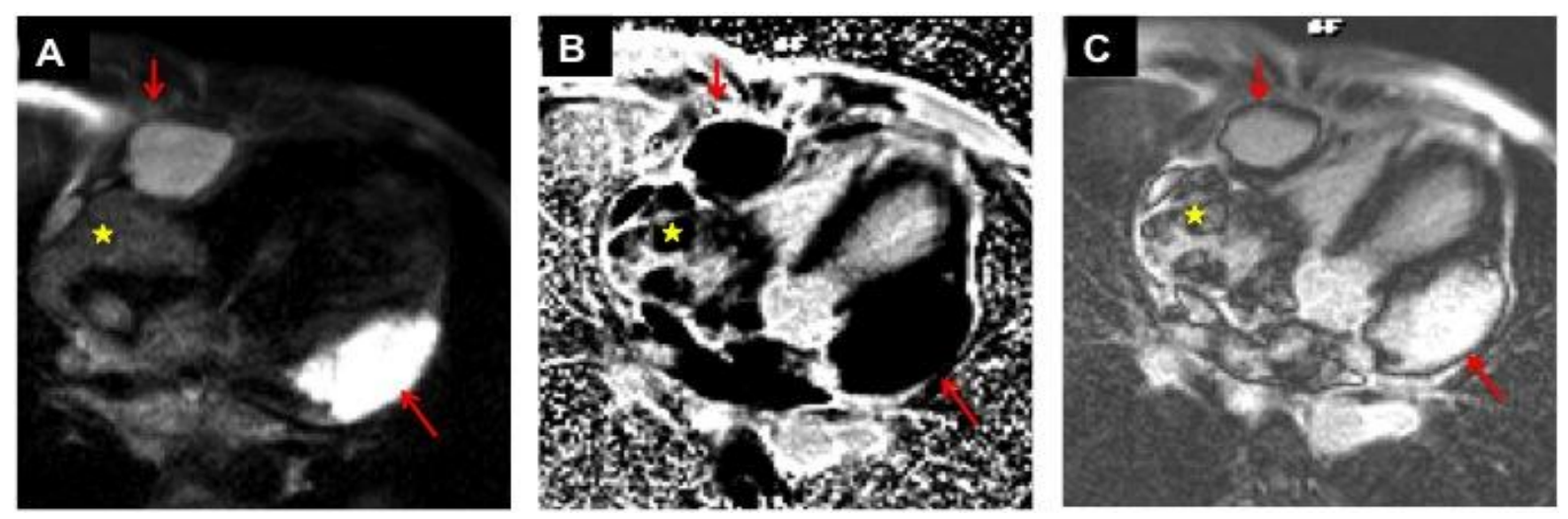

Fig 10: Tubercular mass with pericardial abscesses. (A) T2 W FS 4CH view showing heterogenous intermediate SI mass lesion (yellow star) encasing the RA and two other well circumscribed $\mathrm{T} 2$ hyper intense lesions seen one on right side close to Right AV groove (short red arrow) and the other close to left $\mathrm{AV}$ groove \& lateral surface of $\mathrm{LV}$ (long red arrow). These lesions on post gadolinium images $4 \mathrm{CH}$ view (B and $\mathrm{C}$ ) show peripheral enhancement suggestive of abscess formation. 

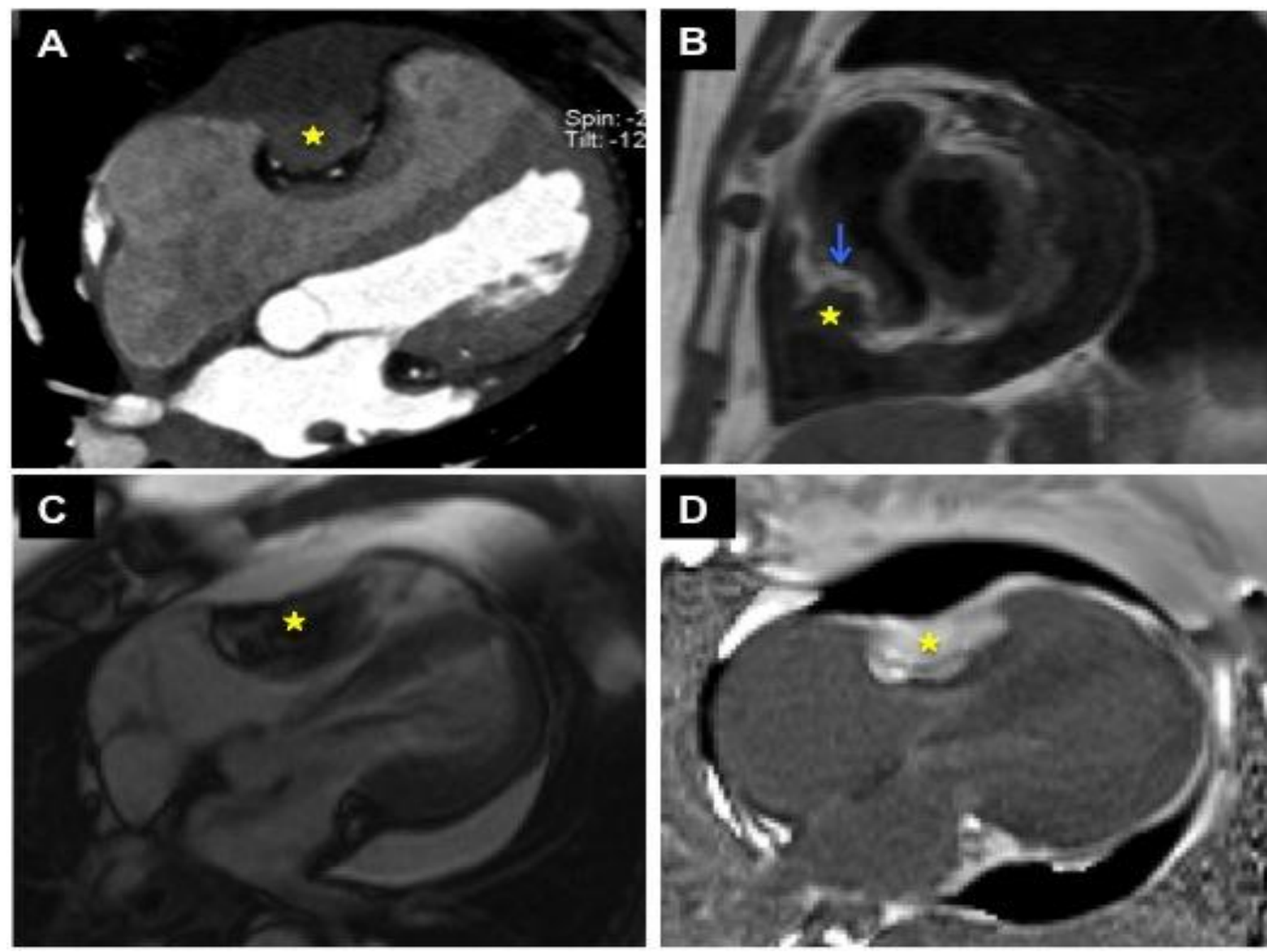

Fig 11: AV Groove pericardial tubercular mass: 34-year-old male with dyspnea on exertion. (A) 4CH CTA image show the heterogeneous mass (yellow star) causing indentation of the Right AVG with preserved epicardial hypo dense fatwhich is also better appreciated in the T1 W MR imageSA view (B) as a Hyper intense (blue arrow) signal and also there is RV surface indentation. (C) 4CH SSFPimage show the hypo to intermediate signal intensity of the mass, which is showing intense enhancement in the post gadolinium image (D). Histopathology showed granulomatous tubercular involvement.

myocardium. The characteristic CMR feature is a homogeneous isointense signal as compared with myocardium on T1 SE and greater hyper intensity on T2 SE (Fig 4). The tumor expands into the pericardial space compressing vessels and cardiac structures; the pericardial invasion by the tumor is identified well with CMR..$^{20,21}$

\section{Lymphoma}

Primary cardiac lymphomas are rare. They occur in the setting of immune suppression and are usually aggressive B- cell lymphoma variants. However, cardiac involvement in lymphoma is usually secondary to disseminated non- Hodgkin's lymphoma and is much more common than primary cardiac lymphoma (Fig 5). Right side of the heart is usual site of involvement with a high predilection for RA. ${ }^{12}$ They may appear isointense or hypointense relative to myocardium on both T1- and T2-weighted FSE sequences and show heterogeneous post gadolinium enhancement. ${ }^{22}$ Common association of pericardial effusion, absence of necrosis, absence of involvement of cardiac valves and intracavitary extension 

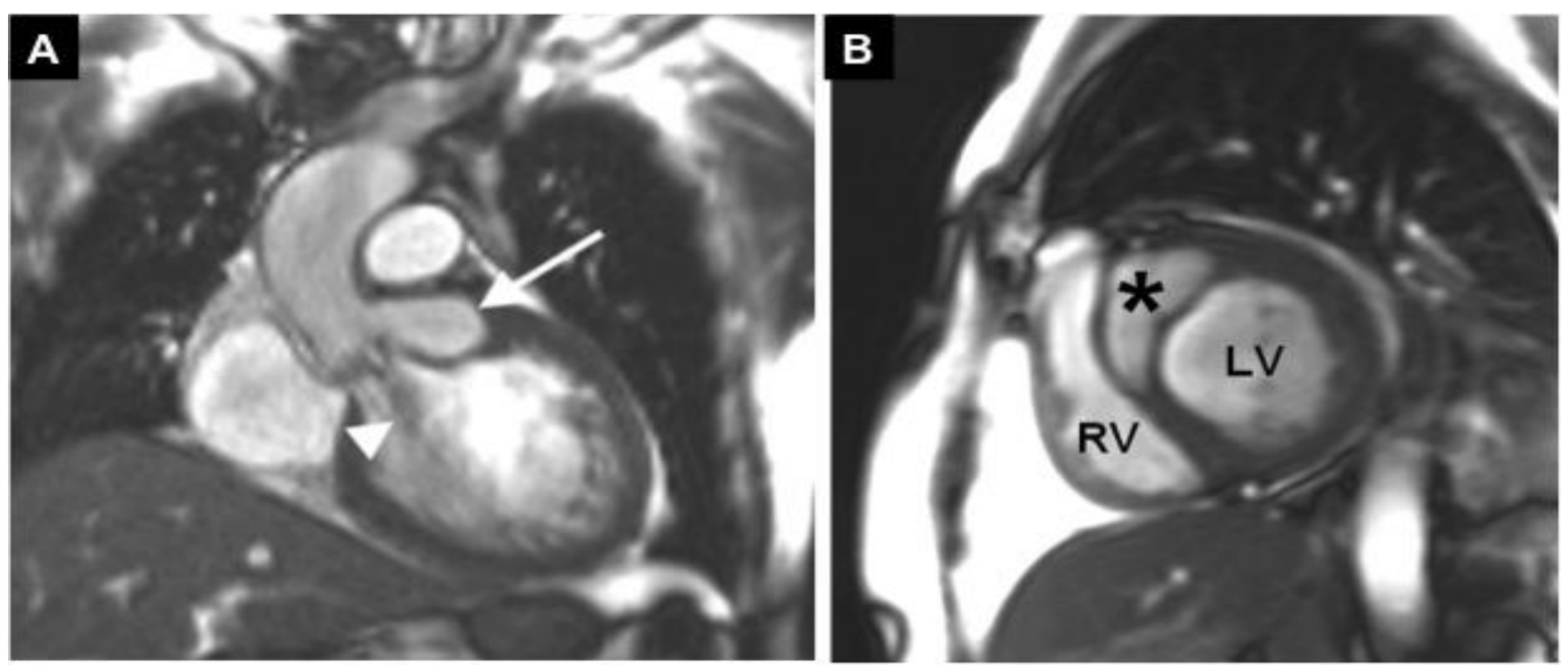

Fig 12: Left sinus of Valsalva aneurysm (SVA) extending into the interventricular septum complicated by aortic regurgitation. (A) Coronal oblique SSFP image in early diastole showing a left SVA extending into the interventricular septum (arrows). Also note aortic central regurgitant jet (arrowhead) and dilated left ventricle (left ventricular end diastolic dimension was $7.2 \mathrm{~cm}$ ). (B) Left ventricular short axis SSFP image at mid ventricular level showing the left SVA dissecting into the interventricular septum (arrows).

are some of the features that help differentiate it from other malignant mass.

\section{Secondary malignant cardiac neoplasm}

\section{Cardiac Metastasis}

Metastases to the heart can be from direct invasion, lymphatic extension, hematogenous spread (Fig 6), or transvenous extension (Fig 7). ${ }^{9} \quad$ Most common manifestation of metastasis is pericardial effusion, which in most of the cases is through lymphatic spread or direct invasion from adjacent structures like lungs, breast and lymph node. The most common cause of cardiac metastasis is from lungs and other common primaries may arise from breast, kidney, and esophagus, as well as lymphoma, leukemia, and melanoma. ${ }^{9}$ Myocardial metastases are often associated with melanoma and lymphoma and are suggestive of hematogenous spread. Renal cell and hepatocellular carcinoma may spread via IVC tumor thrombus into the right atrium. Most metastases show low signal intensity compared with myocardium on T1weighted and high signal intensity on T2weighted SE images, with the exception of melanoma, which appears bright on T1weighted FSE. Nearly all metastasis show post gadolinium enhancement ${ }^{23}$ and hyper perfusion character of these lesions are better appreciated on perfusion imaging ${ }^{13}$.

\section{NON- NEOPLASTIC ETIOLOGY}

\section{Thrombus}

Thrombus appears as a low signal intensity filing defect in the cardiac chamber. Contrast-enhanced CMR allows differentiation between thrombus and surrounding myocardium ${ }^{24}$ because 


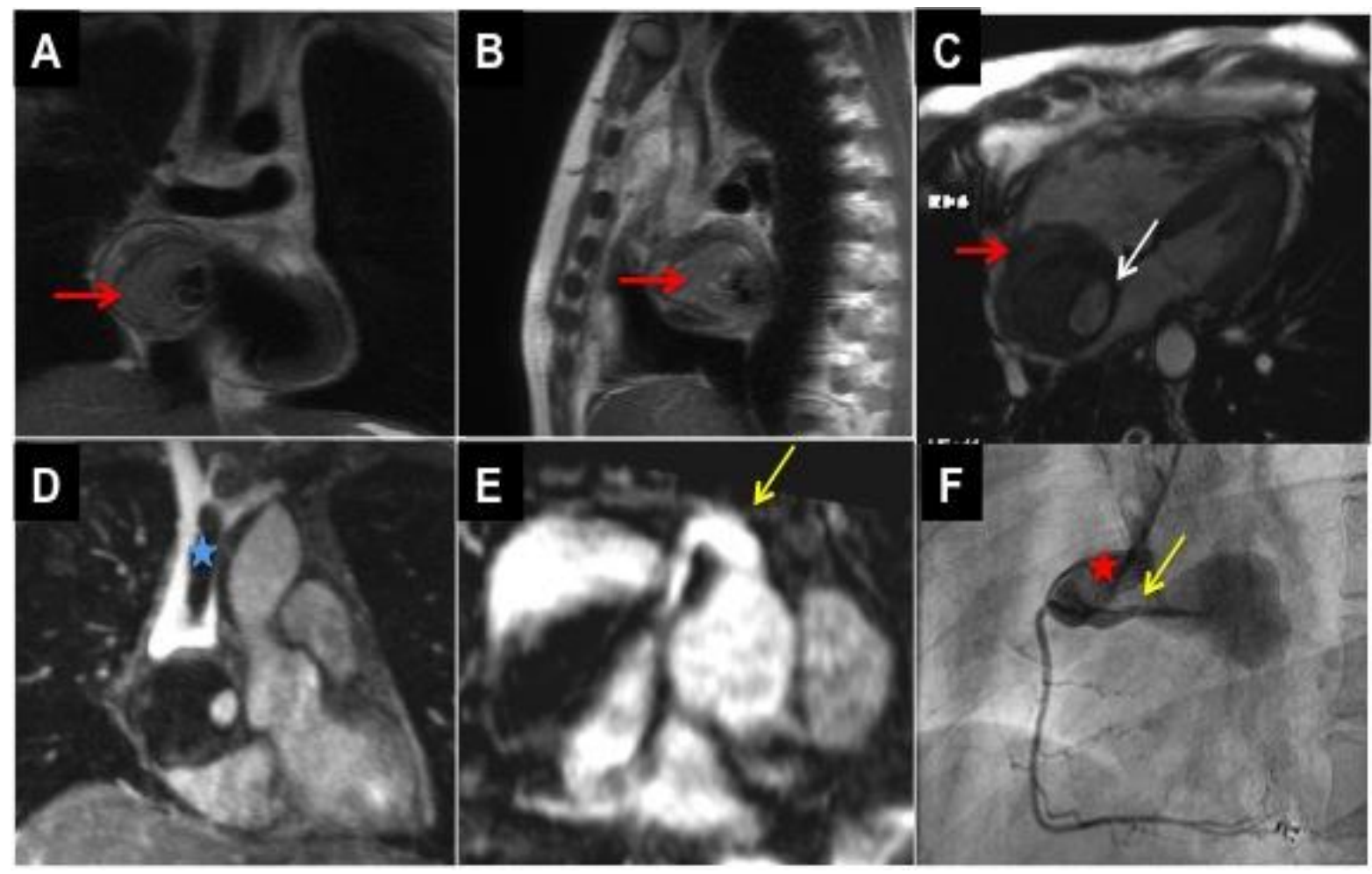

Fig 13: Right coronary artery pseudo aneurysm: (A, B) HASTE coronal and sagittal show a well defined swirled lesion (red arrow) in the right AV groove with signal void at its center and peripheral layering appearance. (C) TRUFI 4CH view show presence of high signal flow (white arrow) within the lesion suggesting the vascular origin (D, E) MIP reformatted coronal and axial oblique view of MR angiogram show that contrast filling of the lesion and the dilated right coronary artery (RCA) (yellow arrow) communicating with the lesion suggestive of RCA pseudo aneurysm. SVC thrombus present (blue star). (F) Catheter angiography image confirms the pseudo aneurysm (red star) arising from the RCA.

thrombus is avascular and hence is Tuberculosis may involve the pericardium characterized by an absence of contrast and or the myocardium. Myocardial uptake. Late gadolinium enhancement involvement is rare with a reported incidence studies are most useful in thrombus detection of $0.3 \%{ }^{27}$ of patients who ide of tuberculosis. as compared to the cine studies (Fig 8). ${ }^{25}$ Three patterns of involvement have been Rarely, large chronic thrombi may enhance described: i) confluent mass (tuberculoma), peripherally, and these cases can be ii) diffuse miliary and iii) diffuse infiltrative. diagnostically challenging. ${ }^{26} \quad$ Right heart chambers are most often affected 27 of these three patterns, the imaging Infective lesions features of tuberculoma are the best described and typically involve single or Cardiac tuberculosis multiple well-defined masses inseparable from the adjacent pericardium. Isolated 
myocardial tuberculomas are extremely rare, usually diagnosed at autopsy. ${ }^{28}$

The tubercular masses usually have iso intense signal character in all sequences. In majority of the cases there is presence of hypo intensity within the lesion on T2W images, which is due to the caseation necrosis. Heterogenous pattern of LGE is seen with non-enhancing areas representing necrosis (Fig 9). ${ }^{29}$ There may be associated pericardial effusion, thickening or pericardial abscess formation (Fig10). Isolated pericardial tubercular involvement may sometimes result in mass formation predominantly involving the atrio ventricular (AV) groove region (Fig 11), with or without myocardial pathology. Although histopathological studies may detect chronic granulomatous inflammatory changes acidfast bacilli stain which are diagnostic of TB are usually negative. Therefore, a combination of history, circumstantial evidence and response to anti tubercular therapy are usually required to provide the diagnosis. ${ }^{30}$ MRI findings with diffuse patterns of involvement have considerable overlap with other forms of myocarditis, with a combination of features suggesting active inflammation and fibrosis. ${ }^{27}$

\section{Infective endocarditis (IE)}

Small vegetations in IE are reliably identified by Transesophageal echocardiography and are difficult to visualize on MRI. Large valvular vegetation may occasionally be seen with MRI, which typically appears as a low-signal mass attached to a valve leaflet, and invariably are associated with valvular regurgitation. The closest differential would be Papillary fibroelastoma, however, valvular regurgitation is rarely seen with fibroelastoma, and it tends to occur away from the valvular free edge. ${ }^{31}$ The main role of cardiovascular MRI in patients of IE is to detect complications of endocarditis, such as aortic root pseudo aneurysms, sinus of Valsalva aneurysms, and embolic vascular lesions.

\section{Cardiac hydatid cyst}

Of all the hydatid locations in the human body cardiac hydatid constitute about 0.5$2 \%$. These can occur on either side of the heart, with right-sided lesions typically expanding the chamber cavity and growing subendocardially, and left-sided lesions more often growing subepicardially. ${ }^{32}$ Secondary cardiac involvement occurs by direct contact with hydatid cysts originating from the liver or lung. Characteristic MRI findings include an oval lesion that is hypo intense on T1W and hyper intense on T2 W. A thin lowintensity peripheral rim on $\mathrm{T} 2$-weighted images, thought to represent dense fibrous capsule from reactive host tissue, is reported to be a characteristic sign of hydatid cyst. ${ }^{32}$

\section{Vascular lesions}

Vascular lesions that can be present as right sided cardiac mass include lesion arising from sinus of valsalva (SOV) (Fig 12), aortic root aneurysms, coronary artery aneurysms (Fig 13) or rarely ventricular aneurysms. SOV arise commonly from right coronary sinus $(72 \%)$ followed by non coronary sinus $(22 \%)$ and rarely left coronary sinus $(6 \%) .{ }^{33}$ These aneurysms can cause mass effect or rupture (66\%). SOV aneurysm most commonly ruptures in to the RV (55.6\%), followed by RA (30.3\%), RVOT (8.5\%), and rarely in to $\operatorname{LV}(2.1 \%) \& \operatorname{LA}(1.1 \%) .{ }^{33}$ 


\section{References}

1. Reynen K. Frequency of primary tumors of the heart. Am J Cardiol. 1996; 77:107.

2. Endo A, Ohtahara A, Kinugawa T, Nawada T, Fujimoto Y, Mashiba $\mathrm{H}$, Shigemasa C. [Clinical incidence of primary cardiac tumors]. J Cardiol. 1996; 28: 227-34.

3. Perchinsky MJ, Lichtenstein SV, Tyers GF. Primary cardiac tumors: Forty years'experience with 71 patients. Cancer. 1997 1; 79: 1809-15.

4. Grande AM, Ragni T, Viganò M. Primary cardiac tumors. A clinical experience of 12 years. Tex Heart Inst J. 1993; 20:22330.

5. Krombach GA, Saeed M, Higgins CB (2003) Cardiac masses. In: Higgins CB, de Roos A (eds) Cardiovascular MRI and MRA. Lippincott Williams \& Wilkins, Philadelphia, PA, pp 136-154.

6. Lam KY, Dickens P, Chan AC. Tumors of the heart. A 20-year experience with a review of 12,485 consecutive autopsies. Arch Pathol Lab Med. 1993; 117(: 102731.

7. Buckley O, Madan R, Kwong R, Rybicki FJ, Hunsaker A. Cardiac masses, part 1: imaging strategies and technical considerations. AJR Am J Roentgenol. 2011; 197:837-41.

8. Hundley WG, Bluemke DA, Finn JP, et al. ACCF/ACR/AHA/NASCI /SCMR 2010 expert consensus document on cardiovascular magnetic resonance: a report of the American College of Cardiology Foundation Task Force on Expert Consensus Documents. Circulation. 2010; 121: 2462-508.

9. Chiles C, Woodard PK, Gutierrez FR, Link KM. Metastatic involvement of the heart and pericardium: CT and MR imaging. Radiographics. 2001; 21:439-49.

10. Araoz PA, MulvaghSsL, Tazelaar HD, Julsrud PR, Breen JF. CT and MR imaging of benign primary cardiac neoplasms with echocardiographic correlation. Radiographics. 2000; 20:1303-19.

11. Kaminaga T, Takeshita T, Kimura I. Role of magnetic resonance imaging for evaluation of tumors in the cardiac region. EurRadiol. 2003; 13:1-10.

12. Luna A, Ribes R, Caro P, Vida J, Erasmus JJ. Evaluation of cardiac tumors with magnetic resonance imaging. EurRadiol. 2005; 15:1446-55.

13. Abdelmoneim SS, Bernier M, Dhoble A, Mankad SV, Mulvagh SL. Assessment of the vascularity of a left atrial mass using myocardial perfusion contrast echocardiography. Echocardiography. 2008; 25:517-20.

14. Donegani G (1972) Tuberous sclerosis. Handbook of clinical neurology, vol 14. North Holland Publishers, Amsterdam, pp 340-349.

15. Grebenc ML, Rosado de Christenson ML, Burke AP, Green CE, Galvin JR. Primary cardiac and pericardial neoplasms: radiologic-pathologic correlation Radiographics. 2000; 20:1073-103.

16. Hananouchi GI, Goff WB. Cardiac lipoma: six- year follow-up with MRI characteristics and a re- view of the literature. MagnReson Imaging 1990; 8:825-828.

17. Gilkeson RC, Chiles C (2003) MR evaluation of cardiac and pericardial malignancy. MagnReson Imaging Clin N Am (1):173-186.

18. Bauner KU, Sourbron S, Picciolo M, et al. MR first pass perfusion of benign and 
malignant cardiac tumours-significant differences and diagnostic accuracy. EurRadiol. 2012; 22:73-82.

19. Yahata S, Endo $\mathrm{T}$, Honma $\mathrm{H}$, et al. Sunray appearance on enhanced magnetic resonance image of cardiac angiosarcoma with pericardial obliteration. Am Heart J. 1994; 127:46871.

20. Gossinger HD, Siostrzonek P, Zangeneh $\mathrm{M}$, et al. Magnetic resonance imaging findings in a patient with pericardial mesothelioma. Am Heart J.1988; 115:1321-2.

21. Vogel HJ, Wondergem JH, Falke TH. Mesothelioma of the pericardium: CT and MR findings. J Comput Assist Tomogr. 1989; 13:543-4.

22. Ryu SJ, Choi BW, Choe KO. CT and MR findings of primary cardiac lymphoma: report upon 2 cases and review. Yonsei Med J. 2001; 42:451-6.

23. O'Donnell DH, Abbara S, Chaithiraphan V, et al. Cardiac tumors: optimal cardiac MR sequences and spectrum of imaging appearances. AJR Am J Roentgenol. 2009; 193:377-87.

24. Barkhausen J, Hunold P, Eggebrecht H, et al. Detection and characterization of intracardiac thrombi on MR imaging. AJR Am J Roentgenol. 2002; 179:153944.

25. Srichai MB, Junor C, Rodriguez LL, et al. Clinical, imaging, and pathological characteristics of left ventricular thrombus: a comparison of contrastenhanced magnetic resonance imaging, transthoracic echocardiography, and transesophageal echocardiography with surgical or pathological validation. Am Heart J. 2006; 152:75-84.

26. Paydarfar D, Krieger D, Dib N, et al. In vivo magnetic resonance imaging and surgical histopathology of intracardiac masses: distinct features of subacute thrombi. Cardiology. 2001; 95:40-7.

27. Khurana R, Shalhoub J, Verma A, et al. Tubercu- lar myocarditis presenting with ventricular tachycardia. Nat ClinPractCardiovasc Med 2008; 5:169174

28. Kapoor OP, Mascarenhas E, Rananaware MM, Gadgil RK. Tuberculoma of the heart. Report of 9 cases. Am Heart J. 1973; 86:334-40.

29. Jagia P, Gulati GS, Sharma S, Goel NK, Gaikwad S, Saxena A. MRI features of tuberculoma of the right atrial myocardium. PediatrRadiol2004; 34: 904-7.

30. Gulati G, Sharma S, Kothari SS, Juneja R, Saxena A, Talwar KK. Comparison of echo and MRI in the imaging evaluation of intracardiac masses. CardiovascInterventRadiol. 2004; 27:459-69.

31. Sparrow PJ, Kurian JB, Jones TR, Sivananthan MU. MR imaging of cardiac tumors. Radiographics2005; 25:1255-76.

32. Dursun M, Terzibasioglu E, Yilmaz R, et al. Cardiac hydatid disease: CT and MRI findings. AJR Am J Roentgenol2008; 190:226-32.

33. Bricker AO, Avutu B, Mohammed TL, et al. Valsalva sinus aneurysms: findings at CT and MR imaging. Radiographics. 2010; 30 :99-110. 